\title{
A 12 MINUTE ORBITAL PERIOD DETACHED WHITE DWARF ECLIPSING BINARY*
}

\author{
Warren R. Brown ${ }^{1}$, Mukremin Kilic ${ }^{1,5}$, J. J. Hermes ${ }^{2}$, Carlos Allende Prieto ${ }^{3,4}$, Scott J. Kenyon $^{1}$, and D. E. Winget ${ }^{2}$ \\ ${ }^{1}$ Smithsonian Astrophysical Observatory, Cambridge, MA 02138, USA; \\ wbrown@cfa.harvard.edu, mkilic@cfa.harvard.edu, skenyon@cfa.harvard.edu \\ 2 Department of Astronomy, University of Texas at Austin, RLM 16.236, Austin, TX 78712, USA; \\ jjhermes@astro.as.utexas.edu, dew@astro.as.utexas.edu \\ ${ }^{3}$ Instituto de Astrofísica de Canarias, E-38205, La Laguna, Tenerife, Spain; callende@iac.es \\ ${ }^{4}$ Departamento de Astrofísica, Universidad de La Laguna, E-38205 La Laguna, Tenerife, Spain \\ Received 2011 June 15; accepted 2011 July 1; published 2011 July 25
}

\begin{abstract}
We have discovered a detached pair of white dwarfs (WDs) with a 12.75 minute orbital period and a $1315 \mathrm{~km} \mathrm{~s}^{-1}$ radial velocity amplitude. We measure the full orbital parameters of the system using its light curve, which shows ellipsoidal variations, Doppler boosting, and primary and secondary eclipses. The primary is a $0.25 M_{\odot}$ tidally distorted helium WD, only the second tidally distorted WD known. The unseen secondary is a $0.55 M_{\odot}$ carbon-oxygen WD. The two WDs will come into contact in 0.9 Myr due to loss of energy and angular momentum via gravitational wave radiation. Upon contact the systems may merge (yielding a rapidly spinning massive WD), form a stable interacting binary, or possibly explode as an underluminous Type Ia supernova. The system currently has a gravitational wave strain of $10^{-22}$, about 10,000 times larger than the Hulse-Taylor pulsar; this system would be detected by the proposed Laser Interferometer Space Antenna gravitational wave mission in the first week of operation. This system's rapid change in orbital period will provide a fundamental test of general relativity.
\end{abstract}

Key words: binaries: close - binaries: eclipsing - gravitational waves - stars: individual (SDSS J065133.338+284423.37) - white dwarfs

Online-only material: color figures

\section{INTRODUCTION}

In Einstein's general theory of relativity, close pairs of stars produce gravitational waves, ripples in the curvature of spacetime (Einstein 1916, 1918). Observations of the Hulse-Taylor binary pulsar PSR B1913+16 confirm the slow decay of the orbit predicted by Einstein's theory and provide indirect evidence for gravitational waves (Hulse \& Taylor 1975; Weisberg et al. 2010). Ongoing and proposed instruments seek to detect gravitational waves directly (Jafry et al. 1994; Hobbs et al. 2009; Abbott et al. 2010). The strongest known gravitational wave sources are compact binaries containing neutron stars and white dwarfs (WDs; e.g., Nelemans 2009). There are presently four known binaries with orbital periods less than 15 minutes - three AM CVn stars (Haberl \& Motch 1995; Israel et al. 1999; Warner \& Woudt 2002) and one low mass X-ray binary (Stella et al. 1987) - but all binaries with $<1 \mathrm{hr}$ orbital periods, with one exception (Kilic et al. 2011b), are interacting systems that complicate direct tests of Einstein's theory. Marsh \& Nelemans (2005) show that the expected period change due to gravitational wave radiation can be modified by both induction-driven angular momentum interchange in magnetic WD systems and by mass transfer in accreting systems. Moreover, there are different explanations for both the nature of the shortest-period AM CVn systems and their observed period changes (Strohmayer 2004; D'Antona et al. 2006; Deloye \& Taam 2006; Roelofs et al. 2010).

Here, we report the discovery of a detached, $765 \mathrm{~s}$ orbital period binary, SDSS J065133.33+284423.3 (hereafter J0651). The discovery comes from our ongoing Extremely Low Mass (ELM)

\footnotetext{
* Based on observations obtained at the MMT Observatory, a joint facility of the Smithsonian Institution and the University of Arizona, and on observations obtained at The McDonald Observatory of The University of Texas at Austin. 5 Spitzer Fellow.
}

WD Survey, a targeted spectroscopic survey for extremely low mass $<0.25 M_{\odot}$ WDs (Brown et al. 2010; Kilic et al. 2010). The ELM Survey demonstrates that extremely low mass WDs are found in compact binaries, and that the majority of these binaries have $<10$ Gyr merger times due to gravitational wave radiation (Kilic et al. 2011a). Depending on the poorly constrained physics of the merger process, these merging WD systems may produce single helium-rich sdO stars, stable mass-transfer AM $\mathrm{CVn}$ binaries, or possibly underluminous supernovae (as discussed in Kilic et al. 2010). The observed merger rate of the low mass WD binaries is about $1 \%$ of the Type Ia supernovae ratecomparable to the rate of underluminous supernovae (Brown et al. 2011). The ELM Survey is also responsible for finding the first tidally distorted WD (Kilic et al. 2011b). J0651 is the most extreme system discovered to date and shows that the ELM Survey may open a new window on gravitational wave sources.

In J0651, the orientation of the binary allows us to observe eclipses of each star by each other, leading to accurate measurement of the orbital parameters, masses, and WD radii. There is no evidence for mass transfer. These results suggest that J0651 is the cleanest known strong gravitational wave source, with an orbital decay time of less than 1 Myr. Future observations will allow us to measure the change in the orbital period. We hope to one day compare this change with direct measurements of gravitational waves and provide an unprecedented test of general relativity.

\section{DATA AND ANALYSIS}

We discovered J0651 on 2011 March 2 as part of our targeted spectroscopic survey for extremely low mass WDs using the 6.5 m MMT telescope. We used the MMT Blue Channel Spectrograph (Schmidt et al. 1989) to obtain $1 \AA$ resolution spectra in the wavelength range $3600 \AA-4500 \AA$. We recognized 


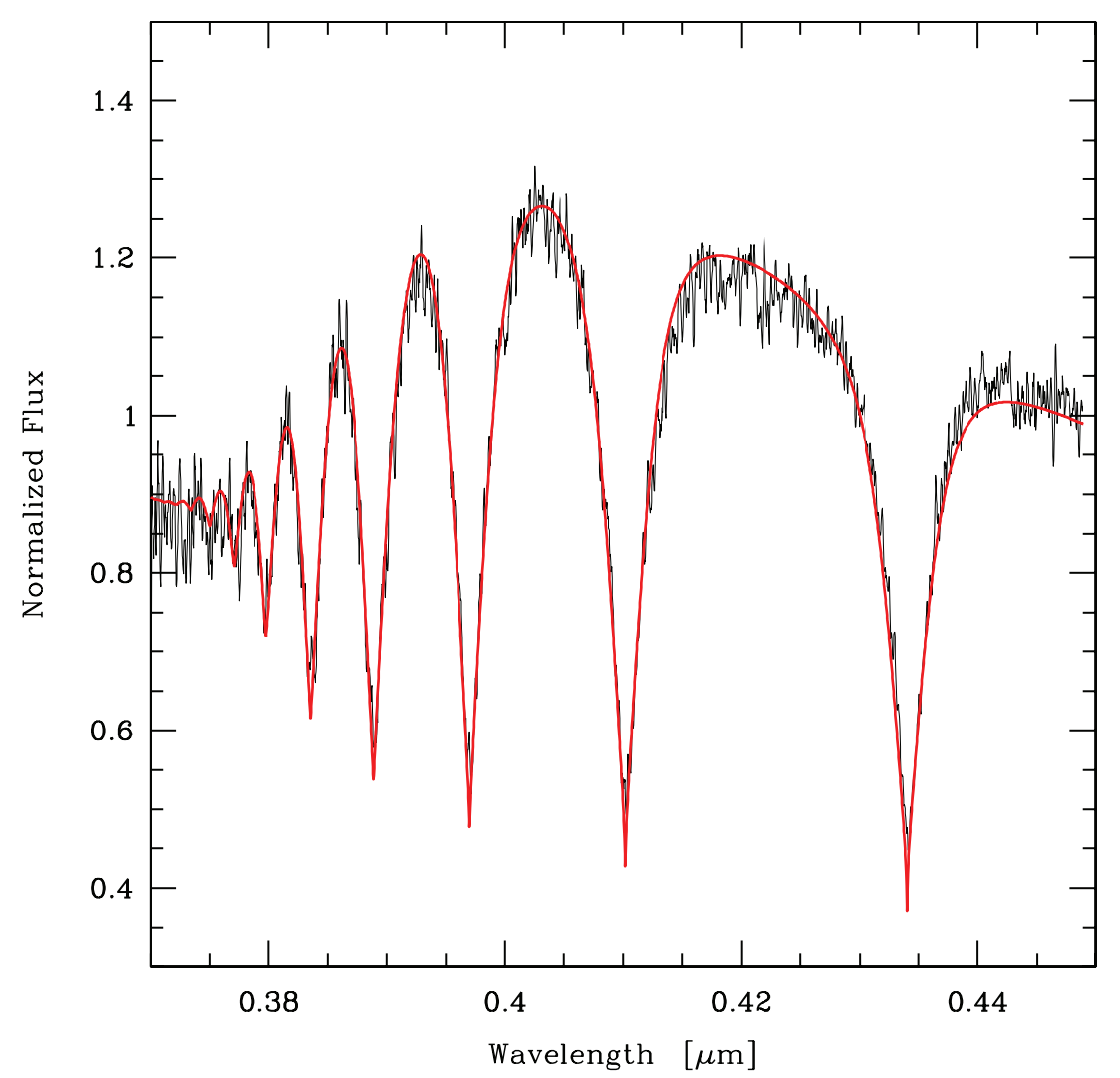

Figure 1. Composite observed spectrum of J0651, constructed by shifting the individual spectra to rest frame and then summing them together to create an effective 60.5 minute exposure, compared to the best-fit stellar atmosphere (solid red line) for a $0.25 M_{\odot}$ WD. The broad hydrogen Balmer absorption lines visually indicate that J0651 is an unusually low mass WD.

(A color version of this figure is available in the online journal.)

J0651 as a low mass WD due to its pressure-broadened hydrogen Balmer lines, which can be seen in Figure 1.

Spectral fits to hydrogen atmosphere WD models (Koester 2008) yield a surface gravity of $\log g=6.79 \pm 0.04$ dex $\left(\mathrm{g}\right.$ in $\left.\mathrm{cm} \mathrm{s}^{-2}\right)$ and an effective temperature of $T_{\text {eff }}=16,400 \pm$ 300 K. The Sloan Digital Sky Survey (SDSS; Aihara et al. 2011) spectrum of this object, on the other hand, yields a systematically larger $\log g=6.97 \pm 0.05$ dex and $T_{\text {eff }}=17,700 \pm 250 \mathrm{~K}$ (S. J. Kleinman 2011, private communication) because the SDSS exposure spans 3.5 orbital periods and thus measures artificially broadened Balmer lines. Our own 6-10 minute exposures of J0651 show similarly overestimated surface gravities and temperatures. A correct understanding of J0651 requires time-resolved spectroscopy only possible with a larger telescope like the $6.5 \mathrm{~m}$ MMT.

Figure 2 compares our best-fit model atmosphere to dereddened $(E(B-V)=0.0706 \mathrm{mag})$ ultraviolet Galaxy Evolution Explorer (GALEX) photometry (Martin et al. 2005), optical SDSS photometry, and our own near-infrared $J=19.599 \pm$ 0.029 mag measurement obtained with SWIRC (Brown et al. 2008) on MMT. The broadband photometry supports our spectroscopic effective temperature and surface gravity measurement with one possible exception. The GALEX near-ultraviolet point shows a $2 \sigma$ discrepancy with the model spectrum convolved with the filter bandpass; however, the photometric error does not include the significant uncertainty in the extinction correction for GALEX filters (Wyder et al. 2005) and so we consider the discrepancy suspect. Comparison with WD evolutionary tracks (Panei et al. 2007) indicates that J0651 is consistent with an extremely low mass $0.25 M_{\odot}$ helium-core WD.
We discovered that J0651 is a compact binary system when back-to-back spectra separated by six minutes showed $\mathrm{a} \simeq 1300 \mathrm{~km} \mathrm{~s}^{-1}$ change in radial velocity (Figure 3 ). Despite the faintness of the $g=19.1 \mathrm{WD}$, the light-gathering power of the $6.5 \mathrm{~m}$ MMT telescope allowed us to reduce our exposure times to 2-2.5 minutes and resolve the orbital period. The data are presented in Table 1 . The observed velocity amplitude is an underestimate, however, because (1) our exposures span $18 \%$ of the orbital phase and (2) the radial velocity curve is sinusoidal, not linear. By integrating a sine curve at the phase covered by our exposures, we determine that the velocity amplitude correction is $5.5 \%$. The observed velocity amplitude is also underestimated if the WD is tidally distorted and its center-of-light differs from its center-of-mass. We do not correct for this effect given that the velocity correction is less than $1 \%$-comparable to our measurement uncertainty - for the observed oblateness.

The best-fit orbital parameters to the radial velocities are: period $P=765.4 \pm 7.9$ s, corrected velocity semi-amplitude $K=657.3 \pm 2.4 \mathrm{~km} \mathrm{~s}^{-1}$, and systemic velocity $\gamma=16.6 \pm$ $0.6 \mathrm{~km} \mathrm{~s}^{-1}$. J0651 is very likely a Galactic disk object based on its small proper motion, $\left(\mu_{\alpha}, \mu_{\delta}\right)=(-3.6,-1.2)$ mas $\mathrm{yr}^{-1}$ (Munn et al. 2004), and small systemic velocity. J0651's location $220 \mathrm{pc}$ above the plane (for a distance of $1 \mathrm{kpc}$, see below) is also consistent with a disk object.

We obtained time-series optical photometry of J0651 with the McDonald Observatory's $2.1 \mathrm{~m}$ Otto Struve Telescope using the ARGOS frame transfer camera (Nather \& Mukadam 2004). Images were obtained with a $B G 40$ Schott glass filter. Our set of $303810 \mathrm{~s}$ and $15 \mathrm{~s}$ exposures comes from five different nights in 2011 April covering a total time baseline of 12.1 days. The 


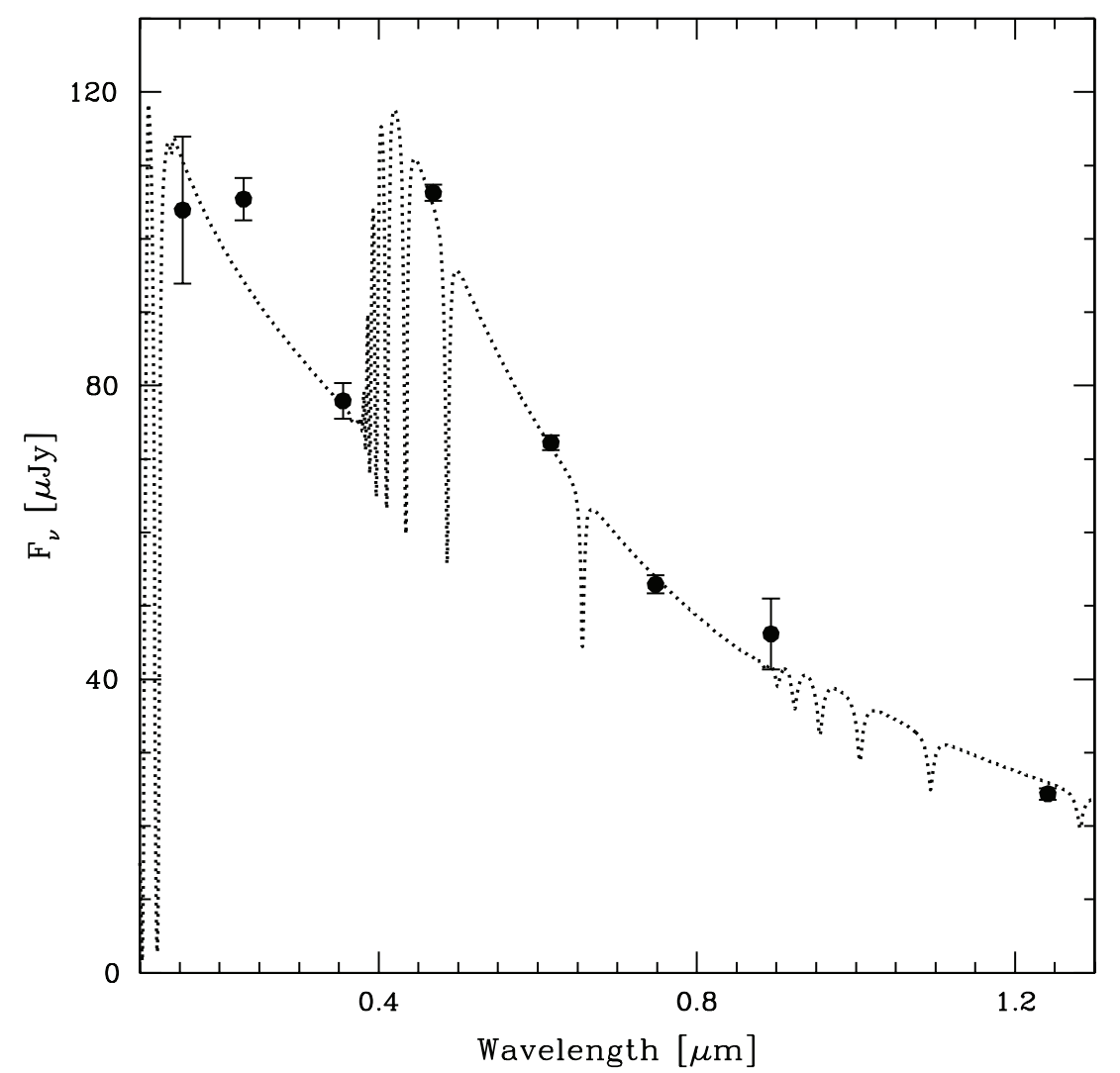

Figure 2. Best-fit WD model atmosphere (dotted line) compared to broadband photometry (dots). The ultraviolet, optical, and near-infrared measurements support our spectroscopic fit for a $0.25 M_{\odot}$ WD.

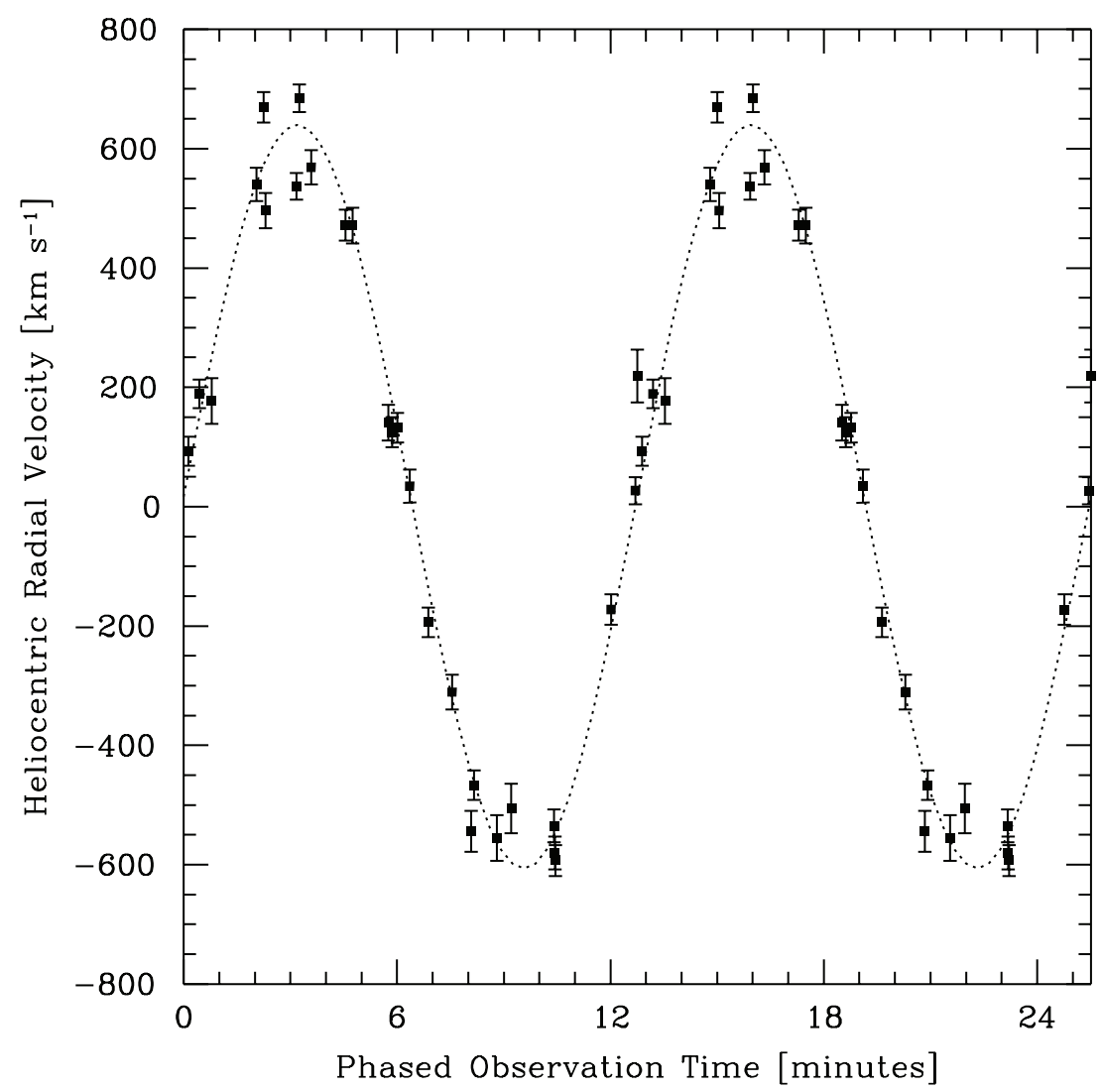

Figure 3. Radial velocity observations phased to the $765 \mathrm{~s}$ orbital period. The best-fit orbit (dotted line) has a $1314.6 \mathrm{~km} \mathrm{~s}^{-1}$ velocity amplitude, which is $0.44 \%$ the speed of light. 


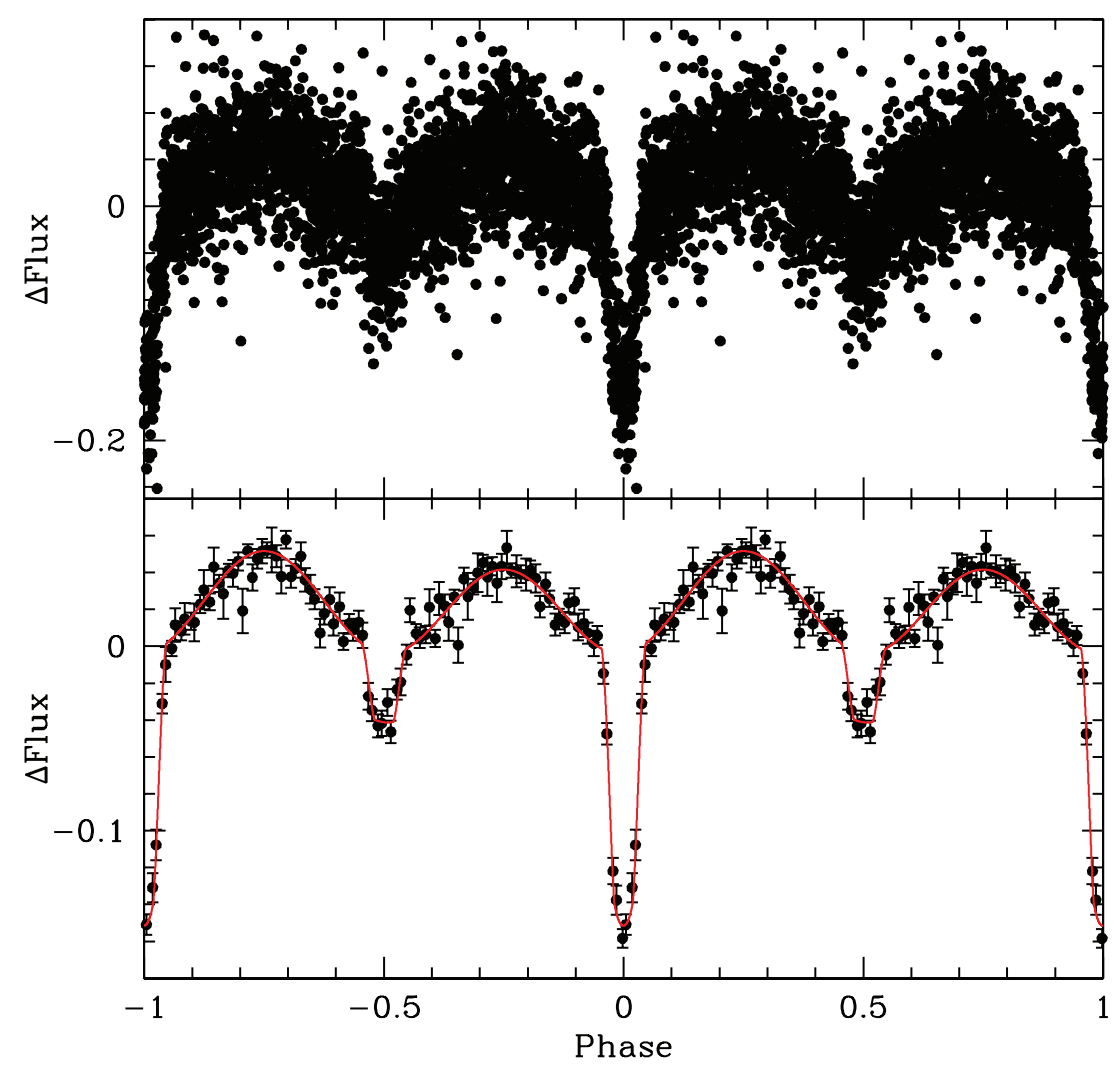

Figure 4. J0651 light curve. The upper panel plots the observed photometry vs. orbital phase, while the lower panel compares the binned data to the best-fit model (solid red line). The data reveal three dramatic features: a sinusoidal pattern due to ellipsoidal variations from the tidally distorted WD, an asymmetric peak in light due to Doppler boosting, and periodic dips in light from the eclipses of the primary (at phase 0) and secondary (at phase 0.5) WDs.

(A color version of this figure is available in the online journal.)

observed light curve, phased to the best-fit period, is plotted in Figure 4 and shows three significant features: a sinusoidal pattern due to ellipsoidal variations from the tidally distorted WD, an asymmetric peak in light due to relativistic beaming (so-called Doppler boosting), and periodic dips in light from the eclipses of the primary and secondary WDs.

We model the light curve of J0651 using JKTEBOP (Southworth et al. 2004) and verify our results with PHOEBE (Prša \& Zwitter 2005). JKTEBOP ${ }^{6}$ and PHOEBE are based on the Eclipsing Binary Orbit Program (Popper \& Etzel 1981) and the Wilson \& Devinney (1971) codes, respectively. We first remove the $0.5 \%$ Doppler boosting (relativistic beaming) signal, however, because neither code models Doppler boosting. Doppler boosting has been seen in only a handful of systems (van Kerkwijk et al. 2010; Mazeh \& Faigler 2010; Shporer et al. 2010; Bloemen et al. 2011); its asymmetric contribution to the J0651 light curve confirms the $765 \mathrm{~s}$ orbital period. We then fit the $5 \%$ amplitude ellipsoidal variations and the $15 \%$ primary eclipse and $4 \%$ secondary eclipse. Reflection effects due to the heating of each WD by its companion are weak, $0.3 \% \pm 0.4 \%$.

The light curve yields a much more precise measurement of orbital period, $P=765.2062 \pm 0.003$ s. The ellipsoidal variations are due to the changing projected area of the distorted primary $\mathrm{WD}$, hence they strongly depend on the inclination

6 JKTEBOP models the projection of each star as a biaxial ellipsoid and calculates a light curve by numerical integration of concentric circles over each star. A by-product of this calculation is an estimate of the oblateness of the primary WD. The $3 \%$ oblateness of J0651 falls within JKTEBOP's $4 \%$ oblateness limit for accurate light curve analysis. angle of the binary system. J0651's ellipsoidal variations and eclipses constrain ${ }^{7}$ the orbital inclination to $i=86.9_{-1.0}^{+1.6}$ degrees.

Eclipses also provide a precise measurement of the WD radii. The $0.25 M_{\odot}$ primary $\mathrm{WD}$ has an observed radius of $0.0353 \pm 0.0004 R_{\odot}$ that differs by $5 \%$ from the $0.0337 R_{\odot}$ radius predicted by helium WD models (Panei et al. 2007). Going in the other direction, the models predict that a WD with the observed radius and with mass $0.24 M_{\odot}$ has $\log g=$ $6.71 \mathrm{dex}$ and $T_{\text {eff }}=16,000 \mathrm{~K}$, consistent with our spectroscopic observations. Given the uncertainties, we consider the observed and predicted radii in excellent agreement.

The unseen secondary has a mass of $0.55 M_{\odot}$ and an observed radius of $0.0132 \pm 0.0003 R_{\odot}$ typical for a carbon-oxygen WD. The eclipse depths indicate that the secondary is $3.7 \% \pm 0.3 \%$ as bright as the primary. Adopting $M_{g}=8.9 \pm 0.1 \mathrm{mag}$ as the absolute magnitude of the $0.25 M_{\odot}$ primary (Panei et al. 2007), the secondary has $M_{g}=12.5 \mathrm{mag}$. A carbon-oxygen WD of this luminosity has $T_{\text {eff }}=9000 \mathrm{~K}, \log g=7.9$ dex, a cooling age of $700 \mathrm{Myr}$, and a radius of $0.0137 R_{\odot}$ (P.-E. Tremblay 2011, private communication). The observed secondary radius differs by $4 \%$ from the predicted radius. There are only two other model-independent mass and radius determinations of helium and carbon-oxygen WDs (Steinfadt et al. 2010; Parsons et al. 2011). Our results thus provide important constraints and reveal an overall agreement with current WD models.

\footnotetext{
7 Errors are estimated from 10,000 Monte Carlo simulations with JKTEBOP
} as described in Southworth et al. (2005). 
Table 1

Radial Velocity Measurements

\begin{tabular}{|c|c|}
\hline $\begin{array}{l}\text { HJD+2455600 } \\
\left(_{\text {days })^{\mathrm{a}}}\right.\end{array}$ & $\begin{array}{c}v_{\text {helio }} \\
\left(\mathrm{km} \mathrm{s}^{-1}\right)\end{array}$ \\
\hline 22.76724617 & $471.0 \pm 30.2$ \\
\hline 22.76919045 & $-310.7 \pm 28.9$ \\
\hline 22.77118102 & $-534.9 \pm 27.8$ \\
\hline 22.77311373 & $189.3 \pm 23.9$ \\
\hline 22.77506958 & $684.5 \pm 23.1$ \\
\hline 22.77697914 & $132.2 \pm 25.0$ \\
\hline 22.77892341 & $-555.3 \pm 38.4$ \\
\hline 22.78221017 & $177.1 \pm 38.3$ \\
\hline 22.78415444 & $568.7 \pm 29.0$ \\
\hline 22.78607558 & $34.5 \pm 27.7$ \\
\hline 22.78806615 & $-505.9 \pm 41.3$ \\
\hline 22.79001043 & $-172.5 \pm 25.4$ \\
\hline 22.79194313 & $540.2 \pm 28.0$ \\
\hline 23.66752771 & $92.8 \pm 24.4$ \\
\hline 23.66963400 & $537.0 \pm 22.3$ \\
\hline 23.67142783 & $140.8 \pm 29.7$ \\
\hline 23.67304806 & $-544.2 \pm 34.5$ \\
\hline 23.67469144 & $-593.0 \pm 26.1$ \\
\hline 23.67628852 & $218.9 \pm 44.2$ \\
\hline 23.67788561 & $496.0 \pm 29.6$ \\
\hline 23.68036224 & $124.7 \pm 25.4$ \\
\hline 23.68195933 & $-466.8 \pm 25.0$ \\
\hline 23.68353327 & $-580.2 \pm 27.4$ \\
\hline 23.68510721 & $26.8 \pm 22.8$ \\
\hline 23.68670429 & $669.3 \pm 25.5$ \\
\hline 23.68830137 & $471.9 \pm 26.0$ \\
\hline 23.68992161 & $-193.7 \pm 24.5$ \\
\hline
\end{tabular}

Note. ${ }^{a}$ Based on UTC.

\section{DISCUSSION}

Having accurately measured J0651's binary parameters, we now calculate the gravitational wave emission predicted by Einstein's general theory of relativity. J0651, with a dereddened apparent magnitude of $g_{0}=18.84 \pm 0.01$ and thus a $1.0 \pm 0.1 \mathrm{kpc}$ distance from the Sun, has a predicted gravitational wave strain (e.g., Roelofs et al. 2007) of $h=1.2 \times 10^{-22}$. J0651 is among the strongest known gravitational wave sources and, more importantly, has an orbital frequency that places it well above the expected gravitational wave foreground (Nelemans 2009). The proposed ESA/NASA Laser Interferometer Space Antenna (LISA) mission has its peak sensitivity at frequencies corresponding to $\simeq 5$ minute orbital periods, and it should detect a strain of $10^{-22}$ at these frequencies with a signal-to-noise ratio of $\simeq 100$ in one year (Roelofs et al. 2007). In other words, J0651 is a verification source that $L I S A$, if built, would detect in the first week of operation.

We also predict that J0651's orbital period is shrinking by $2.7 \times 10^{-4} \mathrm{~s} \mathrm{yr}^{-1}$ due to gravitational wave radiation. The expected change in period adds up to a $5.5 \mathrm{~s}$ change in time-ofeclipse in one year. When we measure this change we expect to provide yet another fundamental test of general relativity and the existence of gravitational waves.

The absence of mass transfer in J0651 is perhaps surprising given how quickly it will merge. In all known binaries with periods comparable to J0651 (e.g., Nelemans 2009), one star fills its Roche lobe and transfers mass to its companion. Our data show that the J0651 primary has a Roche lobe radius 1.5 times its current radius. Under the assumption of energy and angular momentum loss due to gravitational wave radiation, the primary WD will reach its Roche lobe radius at an orbital period of 6.5 minutes in $0.9 \mathrm{Myr}$.

What happens when the primary WD fills its Roche lobe and begins mass transfer in $0.9 \mathrm{Myr}$ is an open theoretical question. The stability of the mass transfer phase depends in part on the known binary mass ratio $q=0.45$ and in part on the unknown tidal synchronization and entropy of the primary WD (Marsh et al. 2004). If mass transfer is stable, J0651 will become an AM CVn system like HM Cancri (Roelofs et al. 2010). Recent WD mass transfer models, on the other hand, predict that mass transfer will cause the pair of WDs to quickly coalesce and merge (Dan et al. 2011). A merger will likely produce a rapidly spinning massive $\mathrm{WD}$, but an underluminous supernova explosion is also a possibility. Underluminous supernovae, such as SN 2005E, are rare types of supernovae that are 10-100 times less luminous than a normal Type Ia supernova and have only $\sim 0.25 M_{\odot}$ worth of ejecta (Perets et al. 2010). The spectrum and light curve of SN 2005E, for example, can be explained by the detonation of a $0.2 M_{\odot}$ helium layer on a $0.45 M_{\odot}$ WD (Waldman et al. 2011) - parameters very similar to the J0651 system. Depending on the nature of the mass transfer, there may be other mechanisms by which the system could detonate (Bildsten et al. 2007; Guillochon et al. 2010). Completing our survey for extremely low mass WD systems such as J0651 will allow us to measure the space density and merger rate of these systems and compare with the rate of underluminous supernovae.

\section{CONCLUSION}

The eclipsing detached WD binary system J0651 presents us with a remarkable laboratory. We can use its eclipses to measure WD masses and radii for detailed tests of WD models. We can use its changing binary orbital period and gravitational wave strain to test for the existence of gravitational waves predicted by general relativity. We can use its very existence to constrain the space density and merger rate of low mass WD binaries and links to underluminous supernovae. In the future, we plan to use multi-passband photometry to directly measure the nature of the secondary WD and to detect the change in orbital period predicted by general relativity.

We thank John Kuehne for his assistance with the observations. This work was supported in part by the Smithsonian Institution and by NASA through the Spitzer Space Telescope Fellowship Program and in part by the National Science Foundation under grant AST-0909107 and the Norman Hackerman Advanced Research Program under grant 003658-0252-2009.

Facilities: MMT (Blue Channel Spectrograph, SWIRC), Struve (Argos)

\section{REFERENCES}

Abbott, B. P., Abbott, R., Acernese, F., et al. 2010, ApJ, 713, 671

Aihara, H., Allende Prieto, C., An, D., et al. 2011, ApJS, 193, 29

Bildsten, L., Shen, K. J., Weinberg, N. N., \& Nelemans, G. 2007, ApJ, 662, L95

Bloemen, S., Marsh, T. R., Østensen, R. H., et al. 2011, MNRAS, 410, 1787

Brown, W. R., Kilic, M., Allende Prieto, C., \& Kenyon, S. J. 2010, ApJ, 723, 1072

Brown, W. R., Kilic, M., Allende Prieto, C., \& Kenyon, S. J. 2011, MNRAS, 411, L31

Brown, W. R., McLeod, B. A., Geary, J. C., \& Bowsher, E. C. 2008, Proc. SPIE, 7014, 70142P

Dan, M., Rosswog, S., Guillochon, J., \& Ramirez-Ruiz, E. 2011, ApJ, submitted (arXiv:1101.5132) 
D’Antona, F., Ventura, P., Burderi, L., \& Teodorescu, A. 2006, ApJ, 653, 1429

Deloye, C. J., \& Taam, R. E. 2006, ApJ, 649, L99

Einstein, A. 1916, Sitzungsberichte der Königlich Preußischen Akademie der Wissenschaften (Berlin: Königlich Preußischen Akademie der Wissenschaften), 688

Einstein, A. 1918, Sitzungsberichte der Königlich Preußischen Akademie der Wissenschaften (Berlin: Königlich Preußischen Akademie der Wissenschaften), 154

Guillochon, J., Dan, M., Ramirez-Ruiz, E., \& Rosswog, S. 2010, ApJ, 709, L64

Haberl, F., \& Motch, C. 1995, A\&A, 297, L37

Hobbs, G. B., Bailes, M., Bhat, N. D. R., et al. 2009, PASA, 26, 103

Hulse, R. A., \& Taylor, J. H. 1975, ApJ, 195, L51

Israel, G. L., Panzera, M. R., Campana, S., et al. 1999, A\&A, 349, L1

Jafry, Y. R., Cornelisse, J., \& Reinhard, R. 1994, ESA J., 18, 219

Kilic, M., Brown, W. R., Allende Prieto, C., et al. 2011a, ApJ, 727, 3

Kilic, M., Brown, W. R., Allende Prieto, C., Kenyon, S. J., \& Panei, J. A. 2010, ApJ, 716, 122

Kilic, M., Brown, W. R., Kenyon, S. J., et al. 2011b, MNRAS, 413, L101

Koester, D. 2008, arXiv:0812.0482

Marsh, T. R., \& Nelemans, G. 2005, MNRAS, 363, 581

Marsh, T. R., Nelemans, G., \& Steeghs, D. 2004, MNRAS, 350, 113

Martin, D. C., Fanson, J., Schiminovich, D., et al. 2005, ApJ, 619, L1

Mazeh, T., \& Faigler, S. 2010, A\&A, 521, L59

Munn, J. A., Monet, D. G., Levine, S. E., et al. 2004, AJ, 127, 3034
Nather, R. E., \& Mukadam, A. S. 2004, ApJ, 605, 846

Nelemans, G. 2009, Class. Quantum Gravity, 26, 094030

Panei, J. A., Althaus, L. G., Chen, X., \& Han, Z. 2007, MNRAS, 382, 779

Parsons, S. G., Marsh, T. R., Gänsicke, B. T., Drake, A. J., \& Koester, D. 2011, ApJ, 735, L30

Perets, H. B., Gal-Yam, A., Mazzali, P. A., et al. 2010, Nature, 465, 322

Popper, D. M., \& Etzel, P. B. 1981, AJ, 86, 102

Prša, A., \& Zwitter, T. 2005, ApJ, 628, 426

Roelofs, G. H. A., Groot, P. J., Benedict, G. F., et al. 2007, ApJ, 666, 1174

Roelofs, G. H. A., Rau, A., Marsh, T. R., et al. 2010, ApJ, 711, L138

Schmidt, G. D., Weymann, R. J., \& Foltz, C. B. 1989, PASP, 101, 713

Shporer, A., Kaplan, D. L., Steinfadt, J. D. R., et al. 2010, ApJ, 725, L200

Southworth, J., Maxted, P. F. L., \& Smalley, B. 2004, MNRAS, 351, 1277

Southworth, J., Smalley, B., Maxted, P. F. L., Claret, A., \& Etzel, P. B. 2005, MNRAS, 363, 529

Steinfadt, J. D. R., Kaplan, D. L., Shporer, A., Bildsten, L., \& Howell, S. B. 2010, ApJ, 716, L146

Stella, L., Priedhorsky, W., \& White, N. E. 1987, ApJ, 312, L17

Strohmayer, T. E. 2004, ApJ, 610, 416

van Kerkwijk, M. H., Rappaport, S. A., Breton, R. P., et al. 2010, ApJ, 715, 51

Waldman, R., Sauer, D., Livne, E., et al. 2011, ApJ, in press (arXiv:1009.3829)

Warner, B., \& Woudt, P. A. 2002, PASP, 114, 129

Weisberg, J. M., Nice, D. J., \& Taylor, J. H. 2010, ApJ, 722, 1030

Wilson, R. E., \& Devinney, E. J. 1971, ApJ, 166, 605

Wyder, T. K., Treyer, M. A., Milliard, B., et al. 2005, ApJ, 619, L15 